\title{
THE NUMBER OF EVEN AND ODD ABSOLUTE PERMUTATIONS OF $\boldsymbol{n}$ LE'T'TERS*
}

BY J. M. THOMAS $†$

A permutation of $n$ letters is called absolute if it leaves no letter fixed. A formula for the total number of such permutations is well known, but no separate formulas for the numbers of even and odd absolute permutations seem to be given in the literature. It is the purpose of this note to show how such formulas can be derived.

Consider the determinant of order $n$

$$
I)=\left|\begin{array}{ccccc}
0 & 1 & 1 & \ldots & 1 \\
1 & 0 & 1 & \ldots & 1 \\
\ldots & \ldots & \ldots & \ldots & \ldots \\
1 & 1 & 1 & \ldots & 0
\end{array}\right|,
$$

all of whose elements are equal to 1 , except those on the main diagonal, which equal 0 . By writing it in the form

$$
\left|\begin{array}{ccccc}
1-1, & 1+0, & 1+0, & \cdots, & 1+0 \\
1+0, & 1-1, & 1+0, & \cdots, & 1+0 \\
\cdots & \cdots & \cdots & \cdots & \cdots \\
1+0, & 1+0, & 1+0, & \cdots, & 1-1
\end{array}\right|
$$

we see that it is expressible as the sum of $2^{n}$ determinants which fall into three classes. Those determinants having more than one column of 1's are zero. Those having exactly one column of 1 's are $n$ in number, and their common value is $(-1)^{n-1}$. Finally there is just one determinant containing no column of 1 's, and its value is $(-1)^{n}$. Hence the value of $D$ is $(-1)^{n-1}(n-1)$.

On the other hand if we consider the expanded form of $D$, say

$$
D=\sum \pm a_{1 i} a_{2 j} \cdots a_{n k},
$$

* Presented to the Society, February 28, 1925.

$\dagger$ National Research Fellow in Mathematics. 
we see that any term which does not correspond to an absolute permutation of the $n$ indices $i, j, \cdots, k$ contains an element from the main diagonal and is therefore zero. The value of a term corresponding to an absolute permutation is +1 or -1 according as the permutation is even or odd. The value of $D$ is therefore the difference between the number of even absolute permutations, $N_{e}$, and the number of odd absolute permutations, $N_{o}$, that is

$$
N_{e}-N_{o}=(-1)^{n-1}(n-1) .
$$

Moreover we have*

$$
N_{e}+N_{o}=n ! \sum_{r=2}^{n} \frac{(-1)^{r}}{r !} .
$$

From (1) and (2) $N_{e}$ and $N_{o}$ can be calculated.

Princeton University

\section{THE ABSOLUTE VALUE OF THE PRODUCT OF TWO MATRICES $\uparrow$}

BY J. H. M. WEDDERBURN

1. Introduction. If $a=\left(a_{p q}\right)$ is a matrix of order $n$ whose elements are ordinary complex numbers, the absolute value of $a$ is defined as $\sqrt{\sum a_{p q} \bar{a}_{p q}}$, where $\bar{a}=\left(\bar{a}_{p q}\right)$ is the matrix whose coefficients are the conjugates of the corresponding coefficients in $a$; we shall denote it here by $\lfloor a\rceil$, a special symbol being convenient since the absolute value of a scalar matrix $\lambda$ is not $|\lambda| \equiv \bmod \lambda$ but $n^{1 / 2}|\lambda|$. This definition has been freely used by writers on differential equations; but, in spite of this, its properties with regard to multiplication have seemingly escaped notice, or are at least not well known.

* Cf. Seelhoff, Archiv der Mathematik und Physik, (2), vol. 1, p. 100.

$\dagger$ Presented to the Society, May 2, 1925. 